\title{
QUASI-COMPLEMENTORS AND COMPLEMENTORS ON CERTAIN BANACH ALGEBRAS
}

\author{
PAK-KEN WONG
}

\begin{abstract}
We study quasi-complementors and complementors on annihilator Banach algebras. We give conditions for a quasi-complementor to be a complementor.
\end{abstract}

1. Introduction. Quasi-complemented algebras, which are generalizations of complemented algebras, were introduced and studied in [5]. Although these two classes of algebras are different in general, they have many similar properties as shown in [5]. In this paper, we continue the study of these algebras.

In \$3, we study some properties of complementors on certain Banach algebras. Let $A$ be a semisimple annihilator Banach algebra with a quasicomplementor $q$. Then we show that $q$ is a complementor on $A$ if and only if, for any orthogonal family of $q$-projections $\left\{f_{\alpha}\right\}$ and $x$ in $A, \sum_{\alpha} f_{\alpha} x$ is summable in the norm of $A$ and especially when $\left\{f_{\alpha}\right\}$ is a maximal family, $x=\Sigma_{\alpha} f_{\alpha} x$. Some particular cases of this result were known, though they appeared in different forms (e.g. see [4] and [7]).

In $\$ 4$, we study continuous complementors. Let $A$ be a $B^{*}$-algebra with a complementor $p$. Then we show that $p$ is continuous if and only if the set $E_{p}$ of all $p$-projections is a closed and bounded subset of $A$. Let $A$ be a semisimple annihilator Banach algebra with a continuous complementor $p$, which has no minimal left ideals of dimension less than three. We show that $A$ is a dense left ideal of some dual $B^{*}$-algebra $B$ and $R^{p}=l(R)^{*} \cap A$ for all closed right ideals $R$ of $A$.

2. Notation and preliminaries. For any subset $S$ in an algebra $A$, let $l_{A}(S)$ and $r_{A}(S)$ denote the left and right annihilators of $S$ in $A$, respectively. Let $A$ be a Banach algebra. Then $A$ is called an annihilator algebra, if for any closed left ideal $J$ and for any closed right ideal $R$, we have $r_{A}(J)=(0)$ if and only if $J=A$ and $l_{A}(R)=(0)$ if and only if $R=A$. If $l_{A}\left(R_{A}(J)\right)=J$ and $r_{A}\left(l_{A}(R)\right)=R$, then $A$ is called a dual algebra.

Received by the editors December 24, 1975 and, in revised form, September 13, 1976 and January 17, 1977.

AMS (MOS) subject classifications (1970). Primary 46C05; Secondary 46K99.

Key words and phrases. Quasi-complementor, complementor, annihilator algebra, dual algebra, q-projection, continuous complementor.

- American Mathematical Society 1977 
Let $A$ be a Banach algebra which is a subalgebra of a Banach algebra $B$. For each subset $S$ of $A, \operatorname{cl}(S)$ (resp. $\operatorname{cl}_{A}(S)$ ) will denote the closure of $S$ in $B$ (resp. $A$ ). Also $l(S)$ and $r(S)$ (resp. $l_{A}(S)$ and $r_{A}(S)$ ) denote the left and right annihilators of $S$ in $B$ (resp. $A$ ). We write $\|\cdot\|$ for the norm on $A$ and $|\cdot|$ for the norm on $B$.

Let $A$ be a Banach algebra and let $I_{r}$ be the set of all closed right ideals in $A$. Following [5], we shall say that $A$ is a (right) quasi-complemented algebra if there exists a mapping $q: R \rightarrow R^{q}$ of $I_{r}$ into itself having the following properties:

$$
\begin{gathered}
R \cap R^{q}=(0) \quad\left(R \in I_{r}\right) ; \\
\left(R^{q}\right)^{q}=R \quad\left(R \in I_{r}\right) ; \\
\text { if } R_{1} \supset R_{2} \text {, then } R_{2}^{q} \supset R_{1}^{q} \quad\left(R_{1}, R_{2} \in I_{r}\right) .
\end{gathered}
$$

The mapping $q$ is called a (right) quasi-complementor on $A . R+R^{q}$ is dense in $A, A^{q}=(0)$ and $(0)^{q}=A$ (see $\left[5\right.$, p. 143]). Hence $R^{q}=(0)$ if and only if $R=A$.

A quasi-complemented algebra $A$ is called a (right) complemented algebra if it satisfies

$$
R+R^{q}=A \quad\left(R \in I_{r}\right)
$$

In this case, the mapping $q$ is called a (right) complementor on $A$ (see $[8, \mathrm{p}$. 651, Definition 1]).

In this paper, all algebras and linear spaces under consideration are over the complex field $C$. Definitions not explicitly given are taken from Rickart's book [7].

3. Quasi-complementors and complementors. Let $\boldsymbol{A}$ be a semisimple Banach algebra with a quasi-complementor $q$. In this section, we find conditions which imply that $q$ is a complementor.

Definition. A minimal idempotent $f$ in a quasi-complemented Banach algebra $A$ is called a $q$-projection if $(f A)^{q}=(1-f) A$. The set of all $q$ projections in $A$ is denoted by $E_{q}$.

LEMMA 3.1. Let $A$ be a semisimple annihilator Banach algebra with a quasicomplementor $q$. Then

(i) Every nonzero right ideal I of $A$ contains a q-projection.

(ii) Let $R$ be a closed right ideal of $A$. If $e$ and $f$ are q-projections contained in $R$ and $R^{q}$, respectively, then $e f=f e=0$.

(iii) If $f_{1}$ and $f_{2}$ are q-projections such that $f_{1} f_{2}=0$, then $f_{2} f_{1}=0$.

Proof. (i) Since $A$ is an annihilator algebra, by [11, p. 37, Lemma 3.1] $I$ contains a minimal right ideal $J$. It follows from the proof of $[5$, p. 149 , Lemma 6.4] that $J$ contains a (unique) $q$-projection which also belongs to $I$.

(ii) Since $e A \subset R,(e A)^{q}=(1-e) A \supset R^{q}$ and so $e f=0$. Similarly $f e=0$. 
(iii) If $f_{1} f_{2}=0$, then $f_{2} \in\left(1-f_{1}\right) A=\left(f_{1} A\right)^{q}$. Thus it follows from (ii) that $f_{2} f_{1}=0$. This completes the proof.

REMARK. Let be a semisimple annihilator quasi-complemented Banach algebra. Then it follows from Zorn's Lemma and Lemma 3.1 that $A$ contains a maximal orthogonal family $\left\{f_{\alpha}\right\}$ of $q$-projections; $A=\operatorname{cl}_{A}\left(\sum_{\alpha} f_{\alpha} A\right)$.

If $A$ is a semisimple complemented Banach algebra, then $x \in \operatorname{cl}_{A}(x A)$ for all $x$ in $A$ (see [2, p. 39, Lemma 3]).

Notation. Let $A$ be a quasi-complemented Banach algebra and $R$ a closed right ideal of $A$. We denote by $P_{R}$ the projection on $R$ along $R^{q}$, i.e., $P_{R}(x+y)=x$ for all $x$ in $R$ and $y$ in $R^{q}$. If $R+R^{q}=A$, then $P_{R}$ is continuous.

THEOREM 3.2. Let $A$ be a semisimple annihilator Banach algebra with a quasicomplementor $q$. Then the following statements are equivalent:

(i) $q$ is a complementor on $A$.

(ii) For any orthogonal family of q-projections $\left\{f_{\alpha}\right\}$ and $x$ in $A, \Sigma_{\alpha} f_{\alpha} x$ is summable in the norm of $A$ and especially when $\left\{f_{\alpha}\right\}$ is a maximal family, $x=\Sigma_{\alpha} f_{\alpha} x$.

Proof. Let $f$ be a $q$-projection, $I$ a closed right ideal and $f \in l_{A}(I)$. Then $I \subset(1-f) A=(f A)^{q}$ and so $f A \subset I^{q}$. With this observation and Lemma 3.1, the proof of Theorem 3.2 now follows exactly like the proof of $[4$, p. 233, Theorem 3.8], with "hermitian idempotent" replaced by " $q$-projection" and " $l_{A}(I) *$ " replaced by " $I^{q}$ ".

Let $\left\{E_{\lambda}: \lambda \in \Lambda\right\}$ be the set of all finite sums $f_{\lambda_{1}}+\cdots+f_{\lambda_{n}}$. For each $\lambda \in \Lambda$, let $P_{\lambda}$ be the projection on $E_{\lambda} A$ along $\left(E_{\lambda} A\right)^{q}$.

Corollary 3.3. If $q$ is a complementor on $A$, then $\left\{\left\|P_{\lambda}\right\|: \lambda \in \Lambda\right\}$ is bounded, where $\left\|P_{\lambda}\right\|$ denotes the operator bound of $P_{\lambda}$.

Proof. Clearly we can assume that $\left\{f_{\alpha}\right\}$ is a maximal family. Then by Theorem 3.2, $x=\sum_{\alpha} f_{\alpha} x$ for all $x$ in $A$. Hence by [4, p. 231, Theorem 3.4], there exists a constant $K$ (independent of $x$ ) such that

$$
\left\|P_{\lambda} x\right\|=\left\|E_{\lambda} x\right\| \leqslant K\|x\| .
$$

Therefore $\left\{\left\|P_{\lambda}\right\|: \lambda \in \Lambda\right\}$ is bounded.

THEOREM 3.4. Let $A$ be a semisimple annihilator Banach algebra which is a dense subalgebra of $a B^{*}$-algebra $B$ such that $|\cdot|$ majorizes $\|\cdot\|$ on $A$ and $x \in \mathrm{cl}_{A}(x A)$ for all $x$ in $A$. Then $A$ is a left ideal of $B$ is and only if the mapping $p: R \rightarrow l(R)^{*} \cap A$ is a complementor on the closed right ideals $R$ of $A$.

Proof. It is well known that $B$ is a dual $B^{*}$-algebra and so the mapping $J \rightarrow l(J)^{*}$ is a complementor on the closed right ideals $J$ of $B$ (see [8, p. 652]). Therefore $p$ is a quasi-complementor on $A$ by the proof of $[5, \mathrm{p}$. 148, Theorem 5.3]. Clearly the set of all hermitian minimal idempotents in $B$ is the set of all $p$-projections. Theorem 3.4 now follows from Theorem 3.2 and the proof of [10, p. 422, Theorem 5.2]. 
For commutative algebras, we have the following stronger version.

Corollary 3.5. Let $A$ be a semisimple commutative complemented Banach algebra with carrier space $X_{A}$. Then $A$ is a dual algebra which is a dense ideal of $C_{0}\left(X_{A}\right)$, the algebra of all complex-valued continuous functions on $X_{A}$ which vanish at infinity.

Proof. Since the socle of $A$ is dense in $A$ [5, p. 143, Lemma 3.3], for every proper closed ideal $R$ of $A$, there exists some minimal idempotent $e$ such that $e \notin R$. Then $e R=(0)$ and so $A$ is an annihilator algebra. Since $x \in \operatorname{cl}_{A}(x A)$ for all $x$ in $A, A$ is a dual algebra by [7, p. 106, Theorem (2.8.29)]. It is easy to see that $A$ is a dense subalgebra of $B=C_{0}\left(X_{A}\right)$ and $\|\cdot\|$ majorizes $|\cdot|$ on $A$. By $[8$, p. 652 , Lemma 1$]$,

$$
R^{p}=l_{A}(R)=l(R) \cap A
$$

for all closed ideals $R$ of $A$. Hence by Theorem 3.4, $A$ is an ideal of $B$.

4. Continuous complementors. Let $A$ be a semisimple annihilator Banach algebra with a complementor $p$ and $M_{A}$ the set of all minimal right ideals of $A$. For each $R \in M_{A}$, by Lemma $3.1, R=f A$ for some $p$-projection $f$ in $A$. Therefore $R+R^{p}=f A+(1-f) A$. Let $P_{R}$ be the projection on $R$ along $R^{p}$. Then $P_{R}$ is continuous. The following definition is given in $[1$, p. 387, Definition].

Definition. Suppose $a_{n} \in A$ with $a_{n} A \in M_{A}(n=0,1,2, \ldots)$. A complementor $p$ on $A$ is said to be continuous if whenever $a_{n}$ converges to $a_{0}$, then $P_{a_{n} A}$ converges to $P_{a_{0} A}$ uniformly on bounded subsets of any minimal left ideal of $A$.

In this section, let $A$ be a semisimple annihilator complemented Banach algebra and $\left\{I_{\lambda}: \lambda \in \Lambda\right\}$ the family of all minimal closed two-sided ideals of $A$. Define $p_{\lambda}$ by $R^{p_{\lambda}}=R^{p} \cap I_{\lambda}$ for all closed right ideals $R$ of $I_{\lambda}$. Then $A$ is the direct topological sum of $\left\{I_{\lambda}: \lambda \in \Lambda\right\}$ and $p_{\lambda}$ is a quasi-complementor on $I_{\lambda}$. Let $H_{\lambda}$ be a minimal left ideal of $I_{\lambda}$. Then $H_{\lambda}$ is a Hilbert space under some equivalent inner product norm. Let $B_{\lambda}$ be the algebra of all completely continuous linear operators on $H_{\lambda}$. Then $I_{\lambda}$ is a dense subalgebra of $B_{\lambda}$ such that $\|\cdot\|$ majorizes $|\cdot|_{\lambda}$ on $I_{\lambda}$, where $|\cdot|_{\lambda}$ is the norm on $B_{\lambda}$ (see [8]).

If $u$ and $v$ are elements of a Hilbert space $H, u \otimes v$ will denote the operator on $H$ defined by the relation $(u \otimes v)(h)=(h, v) u$ for all $h$ in $H$.

LEMMA 4.1. Let $A$ be a simple finite dimensional $B^{*}$-algebra with a complementor $p$ and $E_{p}$ the set of all p-projections in $A$. Then $p$ is continuous if and only if $E_{p}$ is a closed and bounded subset of $A$.

Proof. Let $H$ be a minimal left ideal of $A$ and $E$ the set of all hermitian minimal idempotents in $A$. Then $H$ is a Hilbert space and $A$ can be taken as the $B^{*}$-algebra of all linear operators on $H$. Let $Q$ be the $p$-derived mapping of $p$ (see [3, p. 463, Definition 3.7]). By [1, p. 388, Theorem 2.4], $Q$ is continuous if and only if $p$ is continuous. 
Let $S=\{u \in H:\|u\|=1\}$; then $u \rightarrow u \otimes u$ is a continuous mapping of $S$ onto $E$. If $p$ is continuous, then $u \rightarrow Q(u \otimes u)$ is a continuous mapping of $S$ onto $E_{p}$. Since $H$ is finite dimensional, $S$ is compact and so is $E_{p}$. Thus $E_{p}$ is closed and bounded. The "if" part of the lemma follows from $[9$, p. 257 , Lemma 2].

The following result is similar to [10, p. 257, Theorem 3]. We do not assume any dimension restriction on the minimal left ideals of $A$ here.

THEOREM 4.2. Let $A$ be a $B^{*}$-algebra with a complementor $p$. Then the following statements are equivalent:

(i) The complementor $p$ on $A$ is continuous.

(ii) The set $E_{p}$ of all p-projections is a closed and bounded subset of $A$.

PROoF. Let $E_{p}^{\lambda}$ be the set of all $p$-projections in $I_{\lambda}$; clearly $E_{p}^{\lambda}=E_{p} \cap I_{\lambda}$.

(i) $\Rightarrow$ (ii). Suppose that $p$ is continuous. We show that each $E_{p}^{\lambda}$ is closed and bounded. In fact, this is true if $H_{\lambda}$ is finite dimensional by Lemma 4.1. If $H_{\lambda}$ is infinite dimensional, then by $[1$, p. 395 , Theorem 4.6] and $[9$, p. 257 , Theorem 3], $E_{p}^{\lambda}$ is closed and bounded. Now it is easy to verify that $E_{p}$ is closed (see the proof of [9, p. 257, Theorem 3]). It remains to show that $E_{p}$ is bounded. Suppose this is not so. Then there exists a sequence $f_{n} \in E_{p}^{\lambda_{n}}$ such that $\left\{\left\|f_{n}\right\|\right\}$ is unbounded. Since $f_{m} f_{n} \in I_{\lambda_{m}} \cap I_{\lambda_{n}}=(0)(m \neq n),\left\{f_{n}\right\}$ is an orthogonal family. Therefore by Corollary 3.3, $\left\{P_{f_{n} A}\right\}$ is bounded. Since $B$ is a $B^{*}$-algebra, by $\left[9\right.$, p. 259, Theorem 4] $\left\{f_{n}\right\}$ is bounded, which is a contradiction. Hence $E_{p}$ is bounded and so (ii) follows.

(ii) $\Rightarrow$ (i). Since $E_{p}^{\lambda^{p}}=E_{p} \cap I_{\lambda}(\lambda \in \Lambda)$, by Lemma 4.1 and [3, p. 471, Theorem 6.8] and [1, p. 387, Theorem 2.2], $p$ is continuous. This completes the proof.

ReMARK. "Complementor" cannot be replaced by "quasi-complementor" in Theorem 4.2. In fact, let $B$ and $p$ be given as in [1, p. 396, Example 1]. Then $p$ is a continuous quasi-complementor on $B$, but not a complementor. It is easy to show that $E_{p}$ is unbounded. Since each $P_{n}$ is a continuous complementor on $A_{n}, E_{p}$ is closed.

THEOREM 4.3. Let $A$ be a semisimple annihilator Banach algebra with a continuous complementor $p$. Suppose that $A$ has no minimal left ideals of dimension less than three. Then $A$ is a dense left ideal of some dual $B^{*}$-algebra $B$ and $R^{p}=l(R)^{*} \cap A$ for all closed right ideals $R$ of $A$.

Proof. Let $p_{\lambda}, H_{\lambda}, I_{\lambda}$ and $B_{\lambda}$ be defined as before and ||$_{\lambda}$ the norm on $B_{\lambda}$. By [1, p. 390, Theorem 3.2], $p_{\lambda}$ induces a complementor $q_{\lambda}$ on $B_{\lambda}$ and by [1, p. 391, Theorem 3.3], $q_{\lambda}$ has the form $J_{\lambda}^{q_{\lambda}}=l\left(J_{\lambda}\right)^{*}$ for all closed right ideals $J_{\lambda}$ in $B_{\lambda}$. By [1, p. 393, Lemma 4.3], there exists a constant $M$ such that

$$
\|h\| \leqslant|h|_{\lambda} \leqslant M\|h\| \quad\left(h \in H_{\lambda}, \lambda \in \Lambda\right) .
$$

Let $B$ be the $B^{*}(\infty)$-sum of $\left\{B_{\lambda}: \lambda \in \Lambda\right\}$. Then $B$ is a dual $B^{*}$-algebra. Since $A=\operatorname{cl}_{A}\left(\Sigma_{\lambda} I_{\lambda}\right)$, it follows easily from (4.1) that $A$ can be continuously 
embedded in $B$ and is a dense subalgebra of $B$. Let $H$ be the Hilbert space given in [1, p. 394, Theorem 4.4]. Then by [1, p. 394, Theorem 4.4], we have

$$
R^{p}=\{a \in A: a H \perp R H\}=\left\{a \in A: a^{*} R=(0)\right\} .
$$

Therefore $R^{p}=l(R)^{*} \cap A$ and so by Theorem $3.4, A$ is a left ideal of $B$. This completes the proof.

REMARK 1. Theorem 4.3 shows that there is essentially one type of continuous complementor on $A$.

Remark 2. As shown by an example in [1, p. 396], the dimension restriction in Theorem 4.3 cannot be removed.

The author wishes to thank the referee for his many comments and suggestions.

\section{REFERENCES}

1. F. E. Alexander, Representation theorem for complemented algebras, Trans. Amer. Math. Soc. 148 (1970), 385-398.

2. - On complemented and annihilator algebras, Glasgow J. Math. 10 (1969), 38-45.

3. F. E. Alexander and B. J. Tomiuk, Complemented $B^{*}$-algebras, Trans. Amer. Math. Soc. 137 (1969), 459-480.

4. G. F. Bachelis, Homomorphisms of annihilator Banach algebras, Pacific J. Math. 25 (1968), 229-247.

5. T. Husain and P.-K. Wong, Quasi-complemented algebras, Trans. Amer. Math. Soc. 174 (1972), 141-154.

6. T. Ogasawara and K. Yashinaga, Weakly completely continuous Banach *-algebras, J. Sci. Hiroshima Univ. Ser. A 18 (1954), 15-36.

7. C. E. Rickart, General theory of Banach algebras, Univ. Ser. in Higher Math., Van Nostrand, Princeton, N. J., 1960.

8. B. J. Tomiuk, Structure theory of complemented Banach algebras, Canad. J. Math. 14 (1962), 651-659.

9. P.-K. Wong, Continuous complementors on $B^{*}$-algebras, Pacific J. Math. 33 (1970), 255-260.

10., On the Arens products and certain Banach algebras, Trans. Amer. Math. Soc. 180 (1973), 437-448.

11. B. Yood, Ideals in topological rings, Canad. J. Math. 16 (1964), $28-45$.

Department of Mathematics, Seton Hall University, South Orange, New Jersey 07079 